\title{
Research and design of an Anti Omissions System Based on Single-chip Processor
}

\author{
Xu Jin ${ }^{1}$, Niu Wenyu ${ }^{2}$, Yang $\mathrm{Jie}^{2}$, Chen $\mathrm{Xiaowei}^{3}$ \\ 1Wuhan University of Technology, Wuhan 430000, China
}

Keywords: Single-chip processor, MCS-51 SCM, GSM SMS module

\begin{abstract}
Anti omissions system of nursery school bus based on single-chip processor, which is aimed at resolving security incidents of children omissions. The system consists of microcontroller, infrared microwave double sensors, GSM SMS module and buzzer. It adopted Microwave double infrared, which improve detection accuracy greatly, avoid false reports caused by human factors and natural factors, solve the problem of children left in the school bus to maximum extent, and have broad application prospects.
\end{abstract}

\section{Introduction}

Recently, the accidents of missing children in buses caused by negligence of bus drivers and teachers have occurred frequently. Aiming that, a few places have taken certain measures to prevent, such as installation of infrared in the car, using the IC card and so on. However, because of a mount of false reports caused by human factors and natural factors, it can not achieve social needs well and be unable to resolve the remaining issues. Obviously, preventing children left the car accurately is very important for us.

\section{The overall design of the system}

The system mainly consists of three parts, initiating unit, detecting unit, the response unit. Start unit includes PT2272 remote control transmitter module and PT2262 signal receiver modules (Based on the existing automobiles are used the remote locking manner parked vehicle lock); Detection unit include radar microwave sensors and infrared pyroelectric sensors(Based on the characteristics of a microwave sensor not easily influenced by external temperature or the like, and the characteristics of the infrared sensor detects the human body); Response unit include buzzer and GSM SMS module. The design principle is as follows. Power module is divided into two parts, and one part is used to provide 5V/2A power supply for the GSM module, the other is used to provide $5 \mathrm{~V}$ power supply for the microcontroller; PT2272 signal receiving module detects real-time signals emitted by PT2262 remote control transmitter module, and transfer corresponding level signal to the microcontroller by VT and D0, D1 ports; Pyroelectric infrared sensors and microwave radar sensor detects occupants case and transfer information to the microcontroller through OUT; Microcontroller control GSM SMS module to send a distress message by the commands send by the serial port TXD; MCU control buzzer timely. Diagram of anti omissions system of nursery school bus based on single-chip processor is shown in Figure 1.1. 


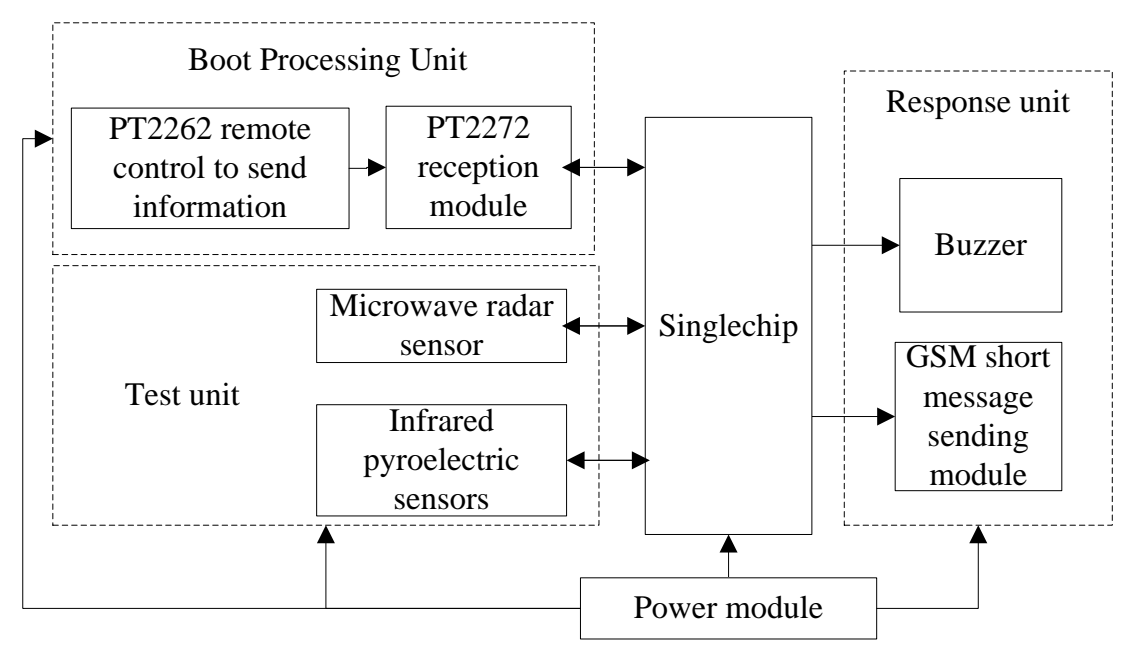

Figure 1.1 System block diagram

System works as follows. Bus driver presses PT2262 remote control transmitter module in the hand to transmit radio signals, when he get off the bus, and then PT2272 signal receiving module receives a radio signal, transfer corresponding level signal to the by D0, D1 ports, microcontroller starts detecting infrared sensor signals from microwave radar sensor module. When the car has staff, infrared sensors and microwave radar sensor module response and send a signal to the microcontroller, and immediately buzzer exude the buzzing sound and GSM module send SMS text messages to drivers. System work flow chart in Figure 1.2

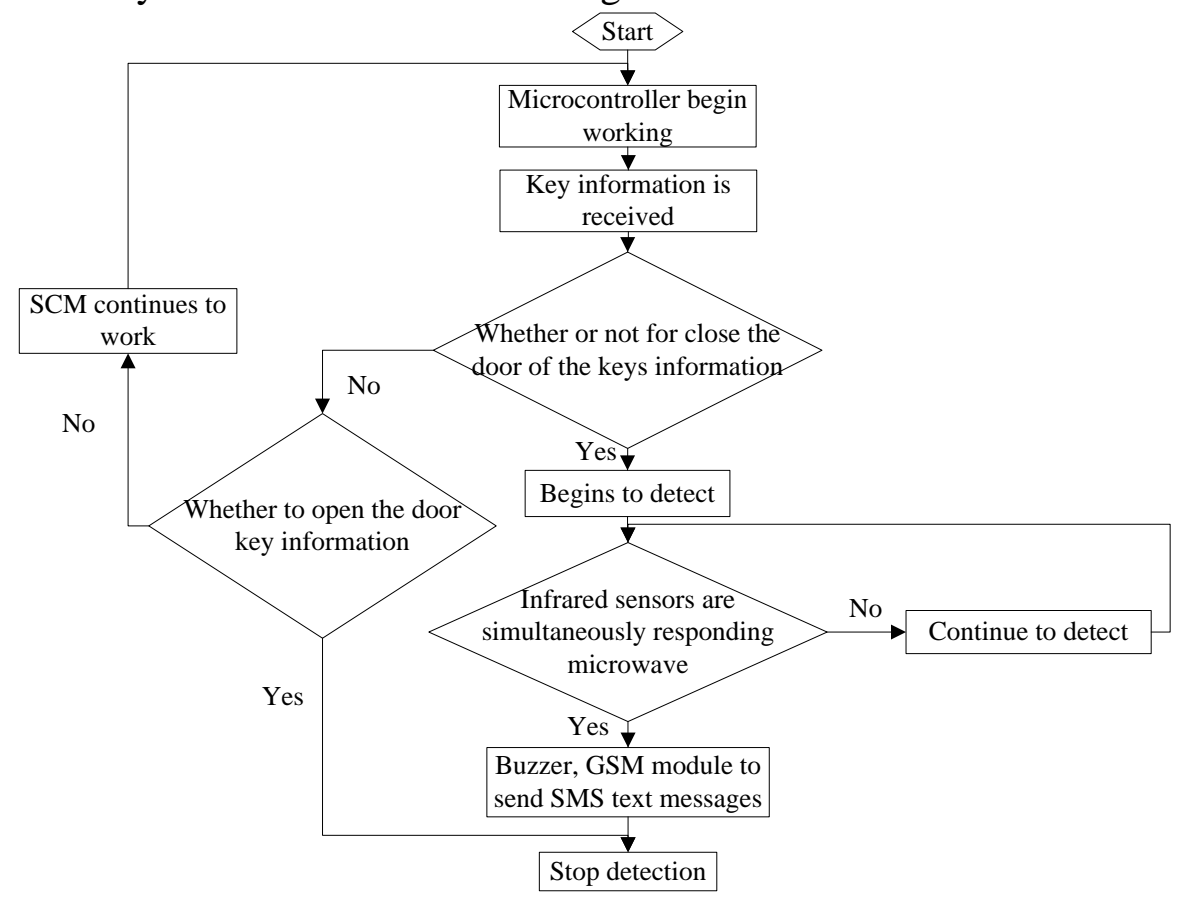

Figure 1.2 System work flow chart

\section{Hardware Design}

Each hardware module's parameter selection. The system's control unit is STC89C51 microcontroller. 4k byte erasable Flash re-flash memory, 128 bytes of internal memory, 5V voltage to provide power, $\mathrm{P} 1$ is a quasi-bidirectional / weak pull-up, and therefore do not require an external pull-up resistor when the I / O port function, Also TXD and RXD for serial communication port.

Use PT2262 remote control transmitter module transmitting radio signals, PT2272 signal receiving module receive wireless signals to determine that the driver door is closed. Transmitter receiver module operating frequency is $315 \mathrm{~Hz}$, receiver module operating voltage is $5 \mathrm{~V}$. Coded transmitter module does not set IC, while add a data modulation transistor Q1. This structure makes it possible to interface easily with other fixed encoding circuit, rolling code circuit and 
microcontroller, regardless of the size of the encoding circuit voltage and the output amplitude of the signal values.

Sensor choose body infrared pyroelectric infrared sensor HC-SR501 and SS-150A microwave radar sensor module. SS-150A uses $12 \mathrm{~V} 100 \mathrm{~mA}$ DC power supply, operating temperature of $-25^{\circ} \sim+50^{\circ}$, the effective detection range is 7 meters, HC-SR501 Operating voltage is DC5V 20V, adjustable range of delay time is $(0.3 \mathrm{~s} \sim 10 \mathrm{~min})$, blocking time is $0.2 \mathrm{~s}$, and there are two kinds of trigger ways including repeated and unrepeated triggering, the sensing range is less than 120 degrees cone angle, 7 meters or less, operating temperature is $-15^{\circ} \sim 70^{\circ}$.

GSM SMS module uses GTM900B, and it supports the MCU, USB transferring TTL directed connection such as computer serial port (232 level) connection and microcontroller. The parameter is introduced as shown in table 1.1

Table 1.1 GSM module parameters is introduced

\begin{tabular}{|c|c|}
\hline Project & Description \\
\hline Product name & SR_GTM900B GPRS Development board (Serial) \\
\hline Major function & Voice calls, SMS transceiver, GPRS data transmission \\
\hline EXT-B+ External Battery & 5 -12V (1A above capacity) Interface: DC005/2.54 Pin \\
\hline Communication interface & $5-12 \mathrm{~V}$ (UART 232: DR9 female 3.3 / 5V TTL: 2.54 Pin \\
\hline Support & $\begin{array}{r}\text { China Mobile, China Unicom (not supported by } \\
\text { telecommunications) }\end{array}$ \\
\hline Standard configuration & Development board +GTM900B Module + Antenna \\
\hline
\end{tabular}

Overall circuit design system. MCU clock circuit uses HC49/S-HC/49SS passive crystal, which connects interface XTAL1 and XTAL2 with other two 30pF ceramic capacitors and its nominal frequency is $11.0592 \mathrm{MHZ}$; Reset circuit using buttons and 10uF electrolytic capacitors series connection; Signal receiver module has seven interface, which is connected to P2.2 VT interface with the microcontroller, the signal is transmitted to the microcontroller. Double sensor connected to $5 \mathrm{~V}$ voltage, the output pins are connected to P1.3 and P1.6 to achieve information transfer between the microcontroller and sensor. The buzzer controlling uses transistor Q8050 switching functions, the base connects to P0.1 port, and a cathode connects to the collector of the speaker, emitter connects to ground. GSM SMS interface module has VCC, GND, TXD, RST and RI ports and VCC, GND, RXD are used. SMS module's power requirements are high, and need 1-2A current to work normally. Overall system circuit diagram is shown in Figure 1.3.

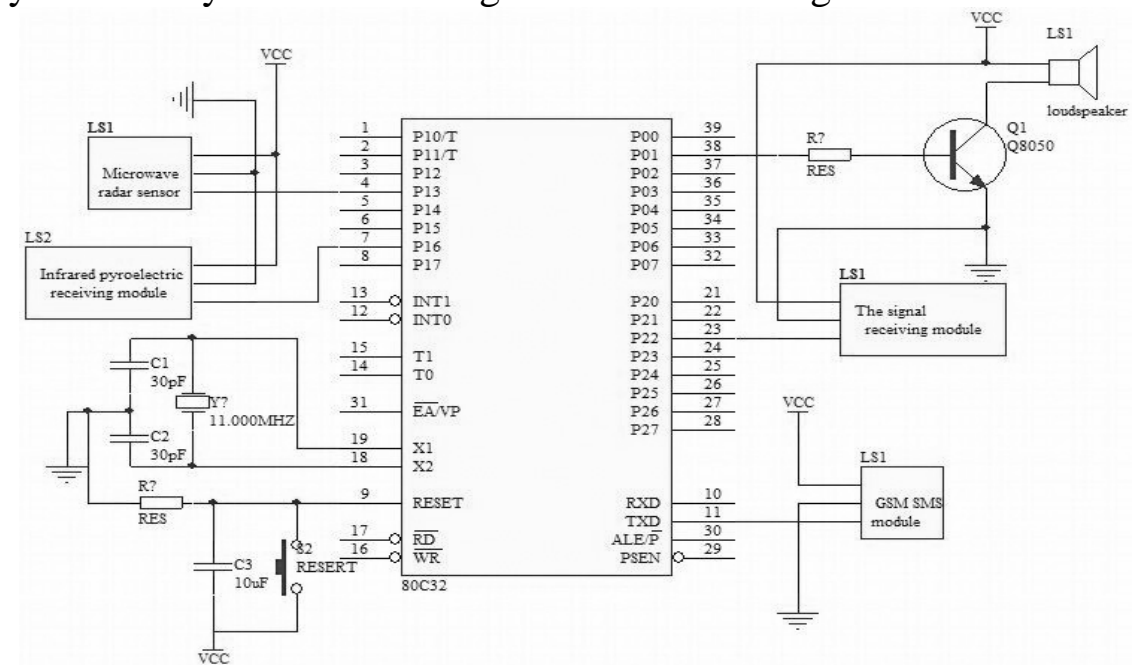

Figure 1.3 Overall system schematic 


\section{Software Design}

System program consists of four parts, extract wireless telemetry data, detection the sensor begins to the transmission of control commands, the microcontroller to obtain sensor information, coding and control buzzer SMS messages. It will elaborate program designing ideas from above four aspects.

Collection of wireless telemetry data mainly set VT as the effective output of the decoder, and its working principle is that firstly, determine whether the VT is high level, if is it, it indicates that a signal is transmitted over the transmission module, and output signal through D1, D0 corresponding port, transfer the signal to the microcontroller, all process is end.

Sensor starts detect transmission of controlling command, and through the first step of data collection results doing judgment, if it is the starting detection signal, the sensor begin to detect.

SCM acquisition sensor detection signal is mainly composed of the signals collected by sensors, a high level is output by the sensor, and then transmitted to the single chip microcontroller, trigger work.

The controlling of buzzer use switching function of transistor Q8050, the base connects to P0.1 port and negative of speakers connects to the collector, emitter connects to ground, and when we need to control the buzzer work, simply pulled P0.1.

Several major commands of controlling GSM module to send SMS text messages by SCM. As follows. AT 、 $\mathrm{AT}+\mathrm{CMGF}=0$ and $\mathrm{AT}+\mathrm{CMGS}=*$, the most important of it is to determine representation digital of "*", and coding of SMS module which send Chinese characters controlled by SCM is the PDU code. The following describes the code of system sending distress content.

Remove the "+" sign of center number of the message, and determine whether the length is an even number, if not, add $\mathrm{F}$ in the end, for example, "8613800546500F", then, do the same processing for the set of phone number. Part processing of SMS is to convert a string to Unicode code, for example, "In danger, please timely rescue, longitude: **, latitude: **", and the Unicode code "9047523053719669FF0C8BF753CA65F662A26551FF0C7ECF5EA64E3AFF1A002A002AFF0C 7EF45EA64E3AFF1A002A002A", and then divide the length of this code is by 2, retain two hexadecimal numbers, referred to as $\mathbf{n}$, assemble the resulting number again with code, referred to as msg, and finally, assemble several converted code. Add the string "11000D91" before the phone number, add 0008A7 after the phone number, finally add msg, the whole string of code referred to as all, then all is done. At first, SCM send "AT" to the SMS module through a serial port TXD, return "OK", then send "AT + CMGS = n", finally send "all" code string, send 1A with hexadecimal, and SMS is end.

\section{Conclusions}

Anti Omissions system of nursery school bus based on single-chip processor, which is use wireless infrared remote sensing technology and double microwave technology can determine whether there are missing persons accurately and notify the relevant personnel to implement the rescue by buzzer with SMS module. It solves the problem of children left in the school bus to maximum extent, and have high feasibility, spread easily.

\section{Acknowledgements}

The research work was supported by National Natural Science Foundation of China under Grant No. 61065009 and Natural Science Foundation of Qinghai Provincial under Grant No. 2011-z-756.

\section{References}

[1] S. Wang,B.C. Khoo,G.R. Liu,G.X. Xu,L. Chen. Coupling GSM/ALE with ES-FEM-T3 for fluid-deformable structure interactions [J]. Journal of Computational Physics, 2014,276. 
[2] Priyantha I. Hathurusingha, Kenneth R. Davey. A predictive model for taste taint accumulation in Recirculating Aquaculture Systems (RAS) farmed-fish - demonstrated with geosmin (GSM) and 2-methylisoborneol (MIB) [J]. Ecological Modelling,2014,291.

[3] Predrag Jovanović,Milan Bjelica. Estimation of GSM base station output power cumulative density function [J]. AEUE - International Journal of Electronics and Communications, 2014,:3-24.

[4] Olivier Ines,Fabienne Degroote,Simon Amiard, Chantal Goubely, Maria E. Gallego,Charles I. White. Effects of XRCC2 and RAD51B mutations on somatic and meiotic recombination in A rabidopsis thaliana [J]. Plant J,2013,746:4-23.

[5] Lohner, Szimonetta,Fekete, Katalin,Marosvölgyi, Tamás,Decsi, Tamás. Gender Differences in the Long-Chain Polyunsaturated Fatty Acid Status: Systematic Review of 51 Publications[J]. Annals of Nutrition \&amp; Metabolism,2013,622:6-35. 